\title{
Mitochondrial DNA copy number can influence mortality and cardiovascular disease via methylation of nuclear DNA CpGs
}

Christina A. Castellani', Ryan J. Longchamps', Jason A. Sumpter ${ }^{1}$, Charles E. Newcomb', John A. Lane², Megan L. Grove ${ }^{3}$, Jan Bressler ${ }^{3}$, Jennifer A. Brody ${ }^{4}$, James S. Floyd ${ }^{4}$, Traci M. Bartz ${ }^{4,5}$, Kent D. Taylor ${ }^{6}$, Penglong Wang ${ }^{7,8}$, Adrienne Tin $^{9}$, Josef Coresh 9 , James S. Pankow ${ }^{10}$, Myriam Fornage ${ }^{3,11}$, Eliseo Guallar ${ }^{9}$, Brian O'Rourke ${ }^{12}$, Nathan Pankratz ${ }^{2}$, Chunyu Liu' ${ }^{13}$, Daniel Levy ${ }^{7,8}$, Nona Sotoodehnia ${ }^{4}$, Eric Boerwinkle ${ }^{3}$ and Dan E. Arking ${ }^{1,12^{*}}$ (D)

\begin{abstract}
Background: Mitochondrial DNA copy number (mtDNA-CN) has been associated with a variety of aging-related diseases, including all-cause mortality. However, the mechanism by which mtDNA-CN influences disease is not currently understood. One such mechanism may be through regulation of nuclear gene expression via the modification of nuclear DNA (nDNA) methylation.

Methods: To investigate this hypothesis, we assessed the relationship between mtDNA-CN and nDNA methylation in 2507 African American (AA) and European American (EA) participants from the Atherosclerosis Risk in Communities (ARIC) study. To validate our findings, we assayed an additional 2528 participants from the Cardiovascular Health Study $(C H S)(N=533)$ and Framingham Heart Study (FHS) $(N=1995)$. We further assessed the effect of experimental modification of mtDNA-CN through knockout of TFAM, a regulator of mtDNA replication, via CRISPR-Cas9.

Results: Thirty-four independent CpGs were associated with mtDNA-CN at genome-wide significance $\left(P<5 \times 10^{-8}\right)$. Meta-analysis across all cohorts identified six mtDNA-CN-associated CpGs at genome-wide significance $\left(P<5 \times 10^{-8}\right)$. Additionally, over half of these CpGs were associated with phenotypes known to be associated with mtDNA-CN, including coronary heart disease, cardiovascular disease, and mortality. Experimental modification of mtDNA-CN demonstrated that modulation of mtDNA-CN results in changes in nDNA methylation and gene expression of specific CpGs and nearby transcripts. Strikingly, the "neuroactive ligand receptor interaction" KEGG pathway was found to be highly overrepresented in the ARIC cohort $\left(P=5.24 \times 10^{-12}\right)$, as well as the TFAM knockout methylation $\left(P=4.41 \times 10^{-4}\right)$ and expression $\left(P=4.30 \times 10^{-4}\right)$ studies.
\end{abstract}

Conclusions: These results demonstrate that changes in mtDNA-CN influence nDNA methylation at specific loci and result in differential expression of specific genes that may impact human health and disease via altered cell signaling.

Keywords: Epigenomics, Mitochondrial DNA, Cardiovascular disease, Mortality

\footnotetext{
* Correspondence: arking@jhmi.edu

${ }^{1}$ McKusick-Nathans Department of Genetic Medicine, Johns Hopkins

University School of Medicine, Baltimore, MD, USA

${ }^{12}$ Division of Cardiology, Department of Medicine, Johns Hopkins University

School of Medicine, Baltimore, MD, USA

Full list of author information is available at the end of the article
}

(c) The Author(s). 2020 Open Access This article is licensed under a Creative Commons Attribution 4.0 International License, which permits use, sharing, adaptation, distribution and reproduction in any medium or format, as long as you give appropriate credit to the original author(s) and the source, provide a link to the Creative Commons licence, and indicate if changes were made. The images or other third party material in this article are included in the article's Creative Commons licence, unless indicated otherwise in a credit line to the material. If material is not included in the article's Creative Commons licence and your intended use is not permitted by statutory regulation or exceeds the permitted use, you will need to obtain permission directly from the copyright holder. To view a copy of this licence, visit http://creativecommons.org/licenses/by/4.0/ The Creative Commons Public Domain Dedication waiver (http://creativecommons.org/publicdomain/zero/1.0/) applies to the data made available in this article, unless otherwise stated in a credit line to the data. 


\section{Background}

Mitochondria are cytoplasmic organelles primarily responsible for cellular metabolism and have pivotal roles in many cellular processes, including aging, apoptosis, and oxidative phosphorylation [1]. Dysfunction of the mitochondria has been associated with complex disease presentation including susceptibility to disease and severity of disease [2]. Mitochondrial DNA copy number (mtDNA-CN), a measure of mitochondrial DNA (mtDNA) levels per cell, while not a direct measure of mitochondrial function, is associated with mitochondrial enzyme activity and adenosine triphosphate production. mtDNA-CN is regulated in a tissue-specific manner and in contrast to the nuclear genome, is present in multiple copies per cell, with the number being highly dependent on cell type $[3,4]$. mtDNA-CN estimates can be derived from DNA isolated from blood and is therefore a relatively easily attainable biomarker of mitochondrial function. Cells with reduced mtDNA-CN show reduced expression of vital complex proteins, altered cellular morphology, and lower respiratory enzyme activity [5]. Variation in mtDNA-CN has been associated with numerous diseases and traits, including cardiovascular disease [6-8], chronic kidney disease [9], diabetes [10, 11], and liver disease $[12,13]$. Lower mtDNA-CN has also been found to be associated with frailty and all-cause mortality [10].

Communication between the mitochondria and the nucleus is bi-directional and it has long been known that crosstalk between nuclear DNA (nDNA) and mtDNA is required for proper cellular functioning and homeostasis $[14,15]$. Specifically, bi-directional crosstalk is essential for the maintenance and integrity of cells $[16,17]$, and interactions between mtDNA and nDNA contribute to a number of pathologies $[18,19]$. However, the precise relationship between mtDNA and the nuclear epigenome has not been well defined despite a number of reports which have identified a relationship between mitochondria and the nuclear epigenome. For example, mtDNA polymorphisms have been previously demonstrated to be associated with nDNA methylation patterns [20], DNA methylation levels of PolgA are associated with mtDNA regulation in embryonic stem cells [21, 22], and hyper- and hypo-methylation of nuclear sites has been observed in mitochondria-depleted cancer cell lines [23]. Additionally, differential DNA methylation in brain tissue and corresponding differential gene expression were observed between strains of mice having identical nDNA, but different mtDNA [18] and reduced mtDNA$\mathrm{CN}$ has been associated with inducing cancer progression via hypermethylation of nuclear DNA promoters [24]. Further, mtDNA-CN has been previously associated with changes in nuclear gene expression [25] including in glioblastoma tumors where modulation of
mtDNA-CN induced changes to both DNA methylation and gene expression of the nuclear genome [26, 27].

Thus, gene expression changes identified as a result of mitochondrial variation may be mediated, at least in part, by nDNA methylation. Further, given that it has been well-established that mtDNA-CN influences a number of human diseases, we propose that one mechanism by which mtDNA-CN influences disease may be through regulation of nuclear gene expression via the modification of nDNA methylation.

To this end, we report the results of cross-sectional analysis of this association between mtDNA-CN and nDNA methylation in 5035 individuals from the Atherosclerosis Risk in Communities (ARIC), Cardiovascular Health Study (CHS), and Framingham Heart Study (FHS) cohorts. Further, to determine the causal direction of the association between mtDNA-CN and nDNA methylation, we present results from experimental knockout as well Mendelian randomization. First, to determine if modification of mtDNA-CN leads to changes to nDNA methylation, we modified mtDNA-CN via three independent stable heterozygous knockouts of the TFAM gene (a regulator of mtDNA replication) followed by assessment of nDNA methylation and gene expression profiles in mtDNA-CN-depleted cell lines. Conversely, to determine if modification of nDNA methylation leads to changes in mtDNA-CN, we used Mendelian randomization with methylation quantitative trail loci (meQTLs) as the instrument variable to assess causality between mtDNA-CN (the outcome) and nuclear methylation (the exposure).

\section{Methods}

Additional methods are available in Additional file 1: Supplementary Methods.

\section{Discovery study analysis \\ The atherosclerosis risk in communities cohort (ARIC)}

The ARIC study is a prospective cohort intended for the study of cardiovascular disease in subjects from four communities across the USA: Forsyth County, NC, northwest suburbs of Minneapolis, MN, Jackson, MS, and Washington County, MD [28]. Sample characteristics are available in Additional file 2: Table S1. Following quality control, 1567 African Americans (AA) and 940 European Americans (EA) were used as a discovery cohort (race was self-identified). Participants for ARIC EA were derived from two existing projects, Brain MRI (81.7\%) and OMICS (18.3\%). DNA was extracted from peripheral blood leukocyte samples from visit 2 or 3 using the Gentra Puregene Blood Kit (Qiagen; Valencia, CA, USA) according to the manufacturer's instructions (www.qiagen.com) and hybridized to the Illumina 
Infinium Human Methylation 450K BeadChip and the Genome-Wide Human SNP Array 6.0.

\section{Estimation of mtDNA-CN from Affymetrix Human SNP 6.0 arrays}

The Affymetrix Genome-Wide Human SNP 6.0 array was used to estimate mtDNA-CN for each participant as previously described [29]. Briefly, mtDNA copy number (mtDNA-CN) was determined utilizing the Genvisis software package (http://www.genvisis.org). Initially, a list of high-quality mitochondrial SNPs were handcurated by employing BLAST to remove SNPs without a perfect match to the annotated mitochondrial location and SNPs with off-target matches longer than $20 \mathrm{bp}$. The probe intensities of the 25 remaining mitochondrial SNPs was determined using quantile sketch normalization (apt-probeset-summarize) as implemented in the Affymetrix Power Tools software. To correct for DNA quality, DNA quantity, hybridization efficiency and other technical artifacts, surrogate variable analysis was applied to the BLAST filtered, GC corrected $\log \mathrm{R}$ ratio (LRR) of 43,316 autosomal SNPs. These autosomal SNPs were selected based on the following quality filters: call rate $>98 \%$, HWE $P$ value $>0.00001$, PLINK mishap for non-random missingness $P$ value $>0.0001$, association with sex $P$ value 0.00001 , linkage disequilibrium pruning $\left(r^{2}<0.30\right)$, maximal autosomal spacing of $41.7 \mathrm{~kb}$. The median of the normalized intensity, LRR for all homozygous calls was GC corrected and used as initial estimates of mtDNA-CN for each sample. The final measure of mtDNA-CN is represented as the standardized residuals from a race-stratified linear regression adjusting the initial estimate of mtDNA-CN for 15 surrogate variables (SVs), age, sex, sample collection site, and white blood cell count. Technical covariates such as DNA quality, DNA quantity, and hybridization efficiency were captured via surrogate variable analysis (SVA) as previously described [7, 30].

\section{Illumina Infinium Human Methylation 450K BeadChip analysis}

The Infinium Human Methylation 450K BeadChip was used to determine DNA methylation profiles from blood for $>450,000$ CpGs across the human genome.

Bisulfite conversion Bisulfite conversion of $1 \mu \mathrm{g}$ genomic DNA was performed using the EZ-96 DNA Methylation Kit (Deep Well Format) (Zymo Research; Irvine, CA, USA) according to the manufacturer's instructions (www.zymoresearch.com). Bisulfite conversion efficiency was determined by PCR amplification of the converted DNA before proceeding with methylation analyses on the Illumina platform using Zymo Research's Universal Methylated Human DNA Standard and Control Primers.
Normalization and quality control Probes included on the list of cross-reactive $450 \mathrm{~K}$ probes as reported by Chen et al. were removed prior to analysis [31]. The cross-reactive target had to match a minimum of 47 bases to be considered cross-reactive. This led to the removal of $\sim 28,000$ probes. Genome studio background correction and BMIQ normalization were performed [32], and the wateRmelon $\mathrm{R}$ package was used to conduct QC filtering [33].

Samples were removed for the following reasons: (1) failed bisulfite conversion, (2) call rate $<95 \%$, (3) sex mismatch using minfi, (4) weak correlation between available genotypes and genotypes on $450 \mathrm{~K}$ array, (5) weak clustering according to sex in MDS plot, (6) PCA analysis identified them as an outlier ( $\geq 4$ SD from mean), (7) failed sex check, (8) sample pass rate $<99 \%$, (9) only sample to pass on a chip. These filtering settings led to the removal of 68 samples in the AA group and 24 samples in the EA group. If samples were run in duplicate, the sample with the lowest missing rate was retained.

Surrogate variable analysis (SVA) SVAs were generated using the package SVA in $\mathrm{R}$ and protecting mtDNA-CN [30].

Control probe principal components in ARIC European Americans The control probe principal components are based on 42 measures, which are transformed from control probes and out-of-band probes in the $450 \mathrm{~K}$ data [34].

\section{Statistical analysis}

All statistical analyses were performed using R (version 3.3.3).

Linear mixed model-association between mtDNA$\mathrm{CN}$ and nuclear DNA methylation Linear mixed effects regression analysis was performed to determine the association between mtDNA-CN and nuclear DNA methylation at specific CpGs (Additional file 3: Table S2).

ARIC AA: Methylation $\sim$ mtDNA-CN + Age + Sex + Site + Visit + Chip Position + Plate + CD8 Count + CD4 Count + B-Cell Count + Monocyte Count + Granulocyte Count + Smoking Status + First 10 Surrogate Variables + Chip (as random effect).

ARIC EA: Same model as ARIC AA but further inclusion of Project (Brain MRI or Omics) as well as the first 10 PCs derived from methylation microarray control probes and the composition of natural killer (NK) cells.

Cell types were imputed using the method of Houseman et al. [35]. All correlations were performed using the Pearson method. 
Global methylation distributions were assessed by a chi-square test to compare observed to expected sitespecific methylation.

Discovery meta-analysis A meta-analysis was performed to combine the results from the individual ARIC AA and EA analyses (Additional file 3: Table S2). This analysis was done using the standard error scheme implemented in Metal [36]. CpGs had to have a $P$ value cutoff of $P<0.05$ in ARIC AA and EA analyses to be included in the meta-analysis. Associations that met genome-wide significance were included in subsequent analyses $\left(P=5.0 \times 10^{-8}\right)$. A total of 100 meta-analysis permutations were also performed (permuted $P=3.94 \times$ $\left.10^{-8}\right)$.

Residual bootstrapping Residual bootstrapping was used to determine the most appropriate genome-wide significance cutoff in ARIC EA and AA cohorts (AA: $P<6.22 \times 10^{-8}$, EA: $P<3.03 \times 10^{-7}$ ) (see Additional file 1: Supplementary Methods). Additionally, the qq plots show minimal inflation in ARIC AA, EA, and metaanalysis (Additional file 1: Fig. S1).

Significant $\mathrm{CpGs}$ with high correlation $\left(\mathrm{R}^{2} \geq 0.6\right)$ were identified as non-independent and the $\mathrm{CpGs}$ with the more significant $P$ value was retained. Highly correlated CpGs were consistent between AA and EA results, specifically these CpGs were cg21051031 and cg03964851 $\left(R^{2}: \quad \mathrm{AA}=0.62, \quad \mathrm{EA}=0.63\right) \quad$ and $\operatorname{cg} 06809544$ and $\operatorname{cg} 13393978\left(R^{2}: A A=0.65, E A=0.70\right)$.

\section{Validation cohorts}

\section{The Cardiovascular Health Study (CHS)}

The CHS is a population-based cohort study of risk factors for coronary heart disease and stroke in adults $\geq 65$ years conducted across four field centers [37]. The original predominantly European ancestry cohort of 5201 persons was recruited in 1989-1990 from random samples of the Medicare eligibility lists; subsequently, an additional predominantly African American cohort of 687 persons was enrolled in 1992-1993 for a total sample of 5888. The validation cohort includes 239 AA participants and 294 EA participants from CHS with mtDNA-CN and 450K methylation derived from the same visit (Race was self-identified, Additional file 2: Table S1).

mtDNA-CN estimation using quantitative PCR mtDNA copy number (mtDNA-CN) was determined utilizing a multiplexed real-time quantitative polymerase chain reaction (qPCR) assay with ABI TaqMan chemistry (Applied Biosystems) as previously described [7] (see Additional file 1: Supplementary Methods). The final measure of mtDNA-CN is represented as the standardized residuals from a race-stratified mixed linear regression adjusting for age, sex, and sample collection site.

Methylation analysis Methylation measurements were performed at the Institute for Translational Genomics and Population Sciences at the Harbor-UCLA Medical Center Institute for Translational Genomics and Population Sciences (Los Angeles, CA). DNA was extracted from Buffy coat fractions and subsequently underwent bisulfite conversion using the EZ DNA Methylation kit (Zymo Research, Irvine, CA). Methylation was then assayed using the Infinium HumanMethylation450 BeadChip (Illumina Inc., San Diego, CA) (see Additional file 1: Supplementary Methods).

Regression analysis CHS was analyzed using linear regression with methylation beta values as the dependent variable and mtDNA-CN as the independent variable. Analyses were adjusted for age, sex, batch, measured white blood cell count, and estimated cell type counts.

\section{The Framingham Heart Study (FHS)}

FHS is a prospective study of individuals from Framingham, Massachusetts [38]. The validation cohort includes 1995 EA participants from FHS with mtDNA-CN and $450 \mathrm{~K}$ methylation derived from the same visit (Race was self-identified, Additional file 2: Table S1).

mtDNA-CN estimation from whole genome sequencing mtDNA copy number (mtDNA-CN) was determined from whole genome sequencing data. Cohort-specific mtDNA-CN residuals were obtained by regressing mtDNA-CN on age, sex, and WBC counts. Mitochondrial DNA copy number was estimated by applying the fastMitoCalc software [39] to harmonize build 37 mappings of TOPMed deep whole genome sequencing data (freeze 5). The estimated mitochondrial copy number is twice the ratio of average mitochondrial sequencing depth to average autosomal sequencing depth. We applied inverse normal transformation to mtDNA$\mathrm{CN}$ residuals.

Methylation analysis DNA extraction, methylation quantification (450K BeadChip), and QC were detailed previously [40]. We obtained lab-specific and cohortspecific DNA methylation residuals by regressing methylation beta values on age, sex, batch effects (plate, col., row), and WBC counts. We applied inverse normal transformation to DNA methylation residuals.

Regression analysis A linear mixed model was applied with inverse normal transformed DNA methylation residuals as the dependent variable and inverse normal 
transformed mtDNA-CN residuals as the independent variable, accounting for family structure.

\section{Power of validation study}

The probability was 5\% (CHS AA), 8\% (CHS EA), and 91\% (FHS EA) that the replication studies would detect a relationship between mtDNA-CN and nDNA methylation equal to the minimal effect size detected in the ARIC meta-analysis at a two-sided 0.0015 significance level (based on 34 independent tests).

\section{Validation and all-cohort meta-analyses}

A meta-analysis was performed of all validation cohorts (FHS EA, CHS EA, CHS AA). We also performed an allcohort meta-analysis (ARIC AA, ARIC EA, FHS EA, CHS EA, CHS AA). Both meta-analyses were performed using the standard error scheme implemented in Metal [36] (Additional file 4: Table S3). Cohort-specific data is available through the respective coordinating centers.

\section{Mendelian randomization meQTL analysis}

meQTLs were identified using MatrixEQTL [41]. Imputed genotypes which were previously derived from ARIC for the relevant participants as well as normalized residuals from our $450 \mathrm{~K}$ methylation dataset were used in regression analysis (see Additional file 1: Supplementary Methods). Metasoft [42] was used for meta-analysis; in addition to the fixed effects (FE) model, a random effects (RE) and Han and Eskin's Random Effects model (RE2) were also used and yielded very similar results.

\section{Mendelian randomization methods}

Independent meQTLs were used for Mendelian randomization (MR). Independence was defined by including SNPs in the same linear model. MR with mtDNA-CN as the outcome and methylation as the exposure was undertaken. meQTLs served as the known relationship of genotype on exposure (methylation) and the results of the linear model, $l m(m t D N A \sim m e Q T L$ SNP + Genotyping PCs [4 for EA, 10 for AA]), were calculated. Power for the MR was calculated using the $\mathrm{YZ}$ association function in mRnd [43].

\section{Phenotype analysis}

We compared methylation at the six validated CpGs to phenotypes that are known to be associated with mtDNA-CN. Phenotypes included prevalent diseases (CHD, CVD) as well as incident diseases (CHD, CVD, mortality). The analysis was performed as follows for each cohort:
A) Prevalent diseases (CHD, CVD): glm (PRVCVD resids (methyl) + AGE + SEX + CENTER + RACE, family = binomial (logit))

B) Incident diseases (CHD, CVD, mortality): coxph (Surv (STime,dead) resids (methyl) + AGE + SEX + CENTER + RACE))

Where resids (methyl) represents methylation adjusted for all relevant covariates from the EWAS. The event adjudication process in ARIC, CHS, and FHS consisted of expert committee review of hospital records, telephone interviews, and death certificates (see Additional file 1: Supplementary Methods).

Results from each of the five individual cohorts were meta-analyzed across cohorts using an inverse weighted standard error method [36] to derive an overall phenotype association for each CpG of interest.

We also assessed the relationship between mtDNA$\mathrm{CN}$ and each positive phenotype at the individual cohort level using logistic regression (glm) for prevalent diseases and cox proportional hazards models for incident diseases adjusted for age, sex, center, and genotyping PCs (4 PCs in EA and 10 PCs in AA). We performed this analysis with and without the inclusion of mtDNA$\mathrm{CN}$-associated CpGs.

\section{CRISPR-CAS9 knockout of TFAM Generation of TFAM knockout}

The stable TFAM CRISPR-Cas9 knockout was generated in HEK293T cells using the Origene TFAM - Human Gene Knockout Kit via CRISPR (catalog number: KN215488) following the manufacturer's protocol. The following sgRNA guide sequence was used to generate the stable TFAM knockout lines: GCGTTTCTCCGAAG CATGTG. Lipofection was conducted using Turbofectin 8.0 (catalog number: TF81001). Puromycin was used for selection at a concentration of $1.5 \mu \mathrm{g} / \mathrm{mL}$. Fluorescenceactivated cell sorting (FACS) was used for single cell sorting and clonal expansion. HEK293T cells were grown in DMEM containing 10\% FBS and 1\% penicillinstreptomycin at $37^{\circ} \mathrm{C}$ and $5 \% \mathrm{CO}_{2}$. Sequencing primers used to confirm the TFAM knockout and proper insertion of the Donor plasmid are as follows: TFAM_Left Forward_Primer_2: AGCGACTGTGGACAACTAGC, GFP_Reverse_Primer_2: TCATCTTGTTGGTCAT GCGG, Puro-Forward_Primer_1: CACAACCTCCCCTT CTACGAG, TFAM_Right_Reverse_Primer_1: CCCCAA ACTCCTTACCTGGG.

DNA/RNA/protein isolation, mtDNA-CN estimation, and qPCR to determine TFAM DNA quantity and TFAM expression as well as Western blotting were performed (see Additional file 1: Supplementary Methods). 


\section{Methylation analysis of TFAM knockout lines}

TFAM KO cell lines were hybridized to the Illumina Infinium EPIC BeadChip at The University of Texas Health Science Center at Houston (UTHealth) [44]. Bisulfite conversion efficiency was reviewed in the laboratory using the Bead Array Controls Reporter (BACR) tool, and Illumina chemistry (sample independent controls) performed within acceptable specifications.

All samples passed with detected CpG (0.01) > 97\%.

EPIC BeadChip analysis was performed using the minfi package [45]. Data was normalized using functional normalization [34], and differential methylation was calculated using the dmpFinder function in minfi (Additional file 5: Table S4).

In the cases where the $\mathrm{CpG}$ from the $450 \mathrm{~K}$ array was not represented on the EPIC array, a CpG surrogate was chosen if there was a nearby CpG within 1000 bp upstream or downstream of the original $\mathrm{CpG}$ that was highly correlated with the original CpG $\left(R^{2} \geq 0.6\right)$ and associated with mtDNA-CN in the ARIC analysis $(P<5 \times$ $\left.10^{-8}\right)$.

\section{RNA sequencing of TFAM knockout lines}

RNA preparation RNA quantification was performed using the Qubit RNA BR Assay (Invitrogen \#Q10211) and Qubit 2.0 Fluorometer. The Agilent BioAnalyzer was used for quality control of the RNA prior to library creation, with a minimum RIN of 8.5. Samples were diluted to $300 \mathrm{ng} / \mu \mathrm{L}$ in $12 \mu \mathrm{L}$ molecular biology-grade water, and then submitted to the Genetic Resources Core Facility for RNA sequencing.

Library preparation and sequencing Illumina's TruSeq Stranded Total RNA kit protocol was used to generate libraries (see Additional file 1: Supplementary Methods).

Primary analysis Illumina HiSeq reads were processed through Illumina's Real-Time Analysis (RTA) software generating base calls and corresponding base call quality scores. CIDRSeqSuite 7.1.0 was used to convert compressed bcl files into compressed fastq files [46].

Secondary analysis Each independent cell line was sequenced twice. RNA sequencing fastq files were pseudoaligned to Genome Reference Consortium Human Build 37 (GRCh37) using Kallisto [47]. A total of 100 bootstraps were performed using Kallisto. The R package Sleuth was used for RNA sequencing analysis [48] (Additional file 6: Table S5). Lane was included as a covariate in the Sleuth model. Differentially expressed genes were defined as those with a $P<0.05$.

\section{Integrated analysis of TFAM knockout methylation and expression}

The linear-gwis method in FAST (genotype mode) was used to collapse TFAM KO methylation data into one gene level $P$ value per gene [49]. These gene-level methylation results were combined with gene-level gene expression results for the same gene using the Fisher $P$ value combination method to generate an integrated gene-level methylation/RNA sequencing $P$ value.

\section{GO/KEGG analysis}

Each CpG was annotated with the nearest gene as defined by the closest gene which harbors the $\mathrm{CpG}$ within $1500 \mathrm{bp}$ of the transcriptional start site and extending to the polyA signal. A bias exists when performing gene set analysis for genome-wide methylation data that occurs due to the differing numbers of $\mathrm{CpG}$ sites profiled for each gene [50]. Due to this, we used gometh for GO and KEGG analysis since it is based off of the goseq method which accounts for this bias [51]. We analyzed our individual ARIC/TFAM datasets as well as our TFAM integrated (meth/expression) dataset. We also combined GO/KEGG results for ARIC, TFAM methylation, and TFAM RNA sequencing using the Fisher $P$ value combination method to generate an overall combined $P$ value for each term. Final $P$ value cutoffs used for each analysis were as follows: ARIC Discovery Meta-Analysis (300 CpGs maximized signal-to-noise, $P=5.24 \times 10^{-12}$ ), TFAM methylation (300 CpGs reflected ARIC cutoff, $\left.P=4.41 \times 10^{-4}\right)$, TFAM expression (169 differentially expressed genes, $\left.P=4.30 \times 10^{-4}\right)$, TFAM integrated (methylation/expression) (188 genes met Bonferroni cutoff, $P=8.77 \times 10^{-6}$ ).

\section{Results}

mtDNA-CN is associated with nuclear DNA methylation at independent genome-wide loci in cross-sectional analysis We performed an epigenome-wide association study (EWAS) in DNA derived from blood for 2507 individuals from the ARIC study, comprised of 1567 African American (AA) and 940 European American (EA) subjects (Additional file 2: Table S1, Additional file 3: Table S2). Thirty-four independent CpGs were significantly associated with mtDNA-CN $\left(P<5 \times 10^{-8}\right)$ in a metaanalysis combining the race groups (Fig. 1, Additional file 1: Fig. S1, Additional file 4: Table S3A) (discovery meta-analysis). This conservative $P$ value cutoff was confirmed by permutation testing. In stratified analysis of ARIC AA and EA participants, we identified 23 and 15 independent $\mathrm{CpGs}$ at epigenome-wide significance, respectively (Additional file 1: Fig. S2, Additional file 4: Table S3B,C). Two CpGs were shared by both race groups (cg26094004 and cg21051031). ARIC AA and EA effect sizes for significant results were strongly 


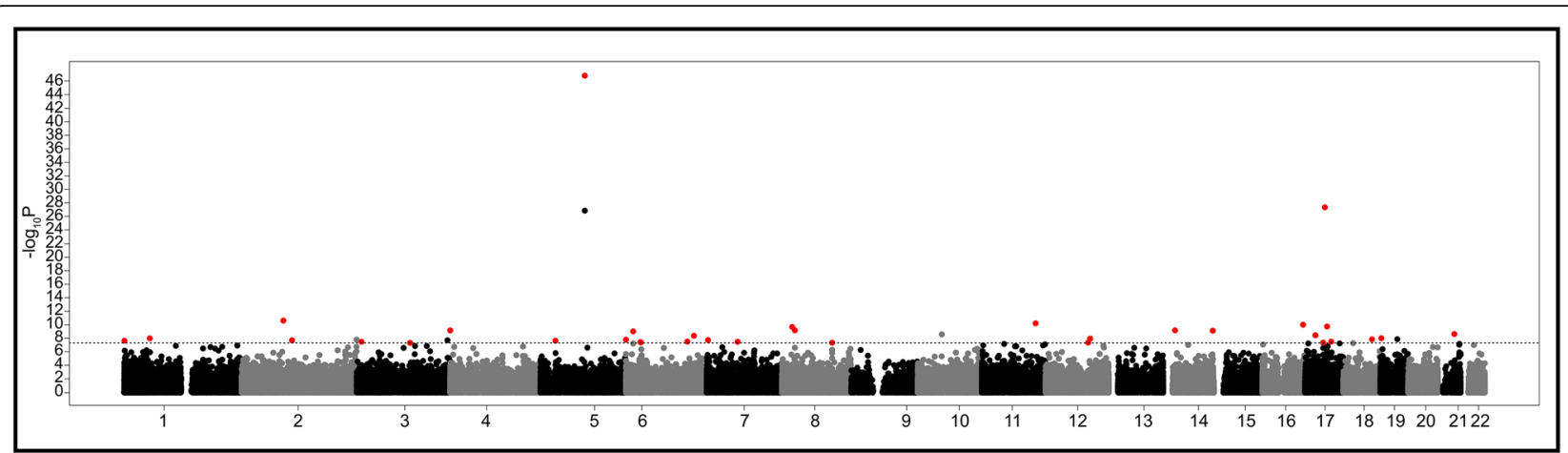

Fig. 1 ARIC discovery meta-analysis (AA and EA) results. In total, 34 independent genome-wide significant CpGs were identified in ARIC metaanalysis to be associated with mtDNA-CN. Dotted line represents genome-wide significance cutoff $\left(P=5 \times 10^{-8}\right)$. CpGs had to be independent and nominally significant in both cohorts $(P<0.05)$, as well as meet the meta-analysis significance cutoff $\left(P=5 \times 10^{-8}\right)$ to be considered significant (red dots). Gray and black dots represents CpGs that were not significant based on this criteria

correlated $\left(R^{2}=0.49\right)$ (Additional file 1: Fig. S3). Further, $16 / 23(70 \%)$ of AA cohort-identified CpGs showed the same direction of effect in EA participants $(P=0.06)$ and 12/14 (86\%) of EA cohort-identified CpGs displayed the same direction of effect in AA participants $(P=0.008)$. Given these observations, we have focused on the ARIC results from combining both races $(N=2507)$ in further analyses. Additionally, an association was observed between increased mtDNA-CN and global hypermethylation $\left(P<2.2 \times 10^{-16}, \beta=0.1487\right)$ in ARIC AA; however, no such association was seen in ARIC EA $(P<0.77, \beta=$ 0.013) (Additional file 1: Fig. S4).

\section{Pathway and biological process analysis displays associations with cell signaling functions and the "neuroactive ligand-receptor interaction" pathway}

To assess the potential mechanism underlying the identified associations, we performed GO and KEGG pathway analysis. mtDNA-CN-associated $\mathrm{CpGs}$ were annotated with their nearest gene. KEGG analysis identified the neuroactive ligand-receptor interaction pathway (path:hsa04080) to be the top overrepresented pathway $\left(P=5.24 \times 10^{-12}\right.$, permuted $\left.P=3.84 \times 10^{-5}\right)$ (Table 1a). Further, GO analyses identified a number of biological processes related to cell signaling and ligand interactions which included cell-cell signaling $\left(P=1.42 \times 10^{-3}\right)$, trans-synaptic signaling $\left(P=1.88 \times 10^{-3}\right)$, and synaptic signaling $\left(P=1.88 \times 10^{-3}\right)$, among others (Table $\left.1 \mathrm{~b}\right)$. These results met permutation tested $P$ value cutoffs.

\section{Validation of CpG associations in independent cohorts}

We performed a validation study to replicate findings from the ARIC discovery population in blood samples from 239 AA and 294 EA participants from CHS as well as 1995 EA participants from FHS, for a total of 2528 individuals (Additional file 2: Table S1). In total, 7/34 CpGs identified in the discovery cohort were nominally significant $(P<0.05$ and same direction of effect as the ARIC cohort results) (Additional file 4: Table S3), and the effect sizes from the ARIC results and the validation meta-analysis were largely correlated $\left(R^{2}=0.36\right)$ (Fig. 2). Overall, the results were consistent across individual cohorts (Additional file 1: Fig. S5, Additional file 4: Table S3) and analysis of the results from the 34 CpGs across all three cohorts (ARIC, CHS, and FHS, $N=5035)$ identified six CpGs as validated mtDNA-CN associated CpGs $\left(P<5 \times 10^{-8}\right)$ (Additional file 4: Table S3, Additional file 1: Fig. S6).

\section{Pathway and biological process analysis of TFAM KO methylation and expression results independently identify pathways observed in cross-sectional analysis}

To assess if modification of mtDNA-CN drives changes to nuclear DNA methylation, we used CRISPR-Cas9 to knock out the TFAM gene, which encodes a regulator of mtDNA replication (Fig. 3). TFAM levels correlate with the level of mtDNA-CN and knockout of TFAM has been shown to reduce mtDNA-CN [52-54]. Stable heterozygous knockout of the TFAM gene (confirmed by qPCR of TFAM DNA) in HEK293T cells resulted in a 5-fold reduction in the steadystate expression levels of TFAM, a marked reduction in protein production $(>81 \%$ ), and an 18 -fold reduction in mtDNA-CN across three independent knockout events (Fig. 4, Additional file 1: Fig. S7). We assayed methylation and expression of genes in the three knockout lines using the Illumina Infinium Methylation EPIC BeadChip and RNA sequencing (Additional file 5: Table S4, Additional file 6: Table S5).

We hypothesized that if similar mechanisms are at play in blood and kidney then consistent pathways would be identified between the cross-sectional cohort analysis and our TFAM KO analysis. We therefore identified overrepresented terms resulting from GO and KEGG analysis of differentially methylated CpGs and differentially expressed genes as well as gene-level integrated methylation and 
Table 1 Results of pathway and functional analysis in ARIC discovery meta-analysis and TFAM knockout methylation and expression datasets with combined $P$ value and ARIC $P$ value $<0.05$. TFAM integrated (INT) $P$ value represents combined methylation and expression results. Combined $P$ value represents combined ARIC and TFAM methylation and expression results

\begin{tabular}{|c|c|c|c|c|c|c|}
\hline & & ARIC $P$ value & $\begin{array}{l}\text { TFAM METH } \\
P \text { value }\end{array}$ & $\begin{array}{l}\text { TFAM RNA } \\
P \text { value }\end{array}$ & $\begin{array}{l}\text { TFAM INT } \\
P \text { value }\end{array}$ & $\begin{array}{l}\text { Combined pathway } \\
\text { P value (ARIC, } \\
\text { TFAM-METH, TFAM-RNA) }\end{array}$ \\
\hline \multicolumn{7}{|c|}{ A. KEGG pathways sorted by combined $P$ value } \\
\hline Pathway & Name & & & & & \\
\hline path:hsa04080 & Neuroactive ligand receptor interaction & $5.24 \mathrm{E}-12$ & 4.41E-04 & 4.30E-04 & 8.77E-06 & $8.96 \mathrm{E}-16$ \\
\hline path:hsa05033 & Nicotine addiction & 8.99E-04 & $6.30 \mathrm{E}-05$ & 9.32E-04 & $7.72 \mathrm{E}-06$ & $1.61 \mathrm{E}-08$ \\
\hline path:hsa04024 & CAMP signaling pathway & $1.29 \mathrm{E}-05$ & $2.32 \mathrm{E}-02$ & $2.23 \mathrm{E}-01$ & $3.25 \mathrm{E}-01$ & 1.03E-05 \\
\hline path:hsa04614 & Renin-angiotensin system & 1.04E-05 & $5.11 \mathrm{E}-02$ & $1.00 \mathrm{E}+00$ & $1.00 \mathrm{E}+00$ & 6.37E-05 \\
\hline path:hsa04723 & Retrograde endocannabinoid signaling & 1.89E-04 & 1.84E-02 & $1.56 \mathrm{E}-01$ & $3.66 \mathrm{E}-03$ & $6.48 \mathrm{E}-05$ \\
\hline path:hsa05032 & Morphine addiction & $1.26 \mathrm{E}-02$ & 4.38E-02 & $5.15 \mathrm{E}-02$ & $1.40 \mathrm{E}-03$ & $1.88 \mathrm{E}-03$ \\
\hline path:hsa05031 & Amphetamine addiction & $3.54 \mathrm{E}-02$ & $9.64 \mathrm{E}-02$ & 4.23E-01 & 1.19E-01 & 4.18E-02 \\
\hline path:hsa04724 & Glutamatergic synapse & 1.80E-02 & $9.49 \mathrm{E}-02$ & $1.00 \mathrm{E}+00$ & $1.00 \mathrm{E}+00$ & 4.73E-02 \\
\hline \multicolumn{7}{|c|}{ B. GO pathways sorted by combined $P$ value } \\
\hline Function & Name & & & & & \\
\hline GO:0007267 & Cell-cell signaling & $1.42 \mathrm{E}-03$ & $1.71 \mathrm{E}-05$ & 1.19E-02 & $1.42 \mathrm{E}-02$ & 7.63E-08 \\
\hline GO:0099537 & Trans-synaptic signaling & $1.88 \mathrm{E}-03$ & 1.06E-05 & $6.27 \mathrm{E}-02$ & $1.92 \mathrm{E}-02$ & $2.89 \mathrm{E}-07$ \\
\hline GO:0099536 & Synaptic signaling & $1.88 \mathrm{E}-03$ & $1.08 \mathrm{E}-05$ & $6.34 \mathrm{E}-02$ & $1.92 \mathrm{E}-02$ & 2.97E-07 \\
\hline GO:0007268 & Chemical synaptic transmission & $1.88 \mathrm{E}-03$ & $2.22 \mathrm{E}-05$ & $6.05 \mathrm{E}-02$ & 1.87E-02 & 5.47E-07 \\
\hline GO:0098916 & Anterograde trans-synaptic signaling & $1.88 \mathrm{E}-03$ & $2.22 \mathrm{E}-05$ & $6.05 \mathrm{E}-02$ & 1.87E-02 & 5.47E-07 \\
\hline GO:0099095 & Ligand-gated anion channel activity & 4.30E-02 & $8.02 \mathrm{E}-04$ & $1.23 \mathrm{E}-04$ & $3.74 \mathrm{E}-07$ & $8.74 \mathrm{E}-07$ \\
\hline GO:0045202 & Synapse & $1.98 \mathrm{E}-04$ & $9.81 \mathrm{E}-05$ & $2.74 \mathrm{E}-01$ & $5.92 \mathrm{E}-03$ & 1.07E-06 \\
\hline GO:0045211 & Postsynaptic membrane & 8.33E-03 & $1.03 \mathrm{E}-04$ & $1.45 \mathrm{E}-01$ & $6.19 \mathrm{E}-04$ & $1.78 \mathrm{E}-05$ \\
\hline
\end{tabular}

expression results (cutoffs used: TFAM Methylation-top 300 differentially methylated CpGs, TFAM Expression-differentially expressed genes (169 genes), TFAM integrated methylation/expression-top 188 genes). The TFAM KO pathway analysis results are consistent with the findings from our ARIC cross-sectional analysis. Specifically, KEGG analysis identified the neuroactive ligand-receptor interaction pathway (path:hsa04080) to be the second most overrepresented pathway in the TFAM knockout methylation analysis $\left(P=4.41 \times 10^{-4}\right)$ and the top overrepresented pathway in the TFAM knockout RNA sequencing analysis $\left(P=4.30 \times 10^{-4}\right)$ (Table 1a). Accordingly, integration of results from TFAM knockout methylation and expression also resulted in strong association with this pathway $(P=$ $8.77 \times 10^{-6}$ ). Further, combining of $P$ values (Fisher's method) across the ARIC meta-analysis, TFAM knockout methylation, and TFAM knockout expression analyses yielded a combined $P$ value of $8.96 \times 10^{-16}$ for this pathway which was also the top pathway identified in integrated analysis (Table 1a).

The specific genes identified by each analysis to be part of the neuroactive ligand receptor interaction pathway were unique to each study (Additional file 2: Table S6), with only one gene (GABRG3) in common between
ARIC analyses and TFAM knockout methylation analysis and only one gene (GABRB1) in common between TFAM knockout methylation and expression analyses (Additional file 2: Table S6).

GO analyses of TFAM knockout cell lines also confirmed the finding from cross-sectional analysis that biological processes related to cell signaling and ligand interactions including cell-cell signaling (combined $P=$ $7.63 \times 10^{-8}$ ), trans-synaptic signaling (combined $P=$ $2.89 \times 10^{-7}$ ), and synaptic signaling (combined $P=$ $2.97 \times 10^{-7}$ ) were overrepresented, among others (Table 1b). These results suggest that mtDNA-CN drives changes to nDNA methylation at sites nearby genes relating to cell signaling processes which in turn may cause gene expression changes to these genes and contribute to disease.

\section{mtDNA-CN is causative of changes in nuclear DNA methylation and nuclear gene expression}

Although we do not a priori expect site-specific methylation results to be consistent with those we have identified in blood due to the fact that HEK293T lines represent a different tissue and are subject to the inherent variability of cell culture 


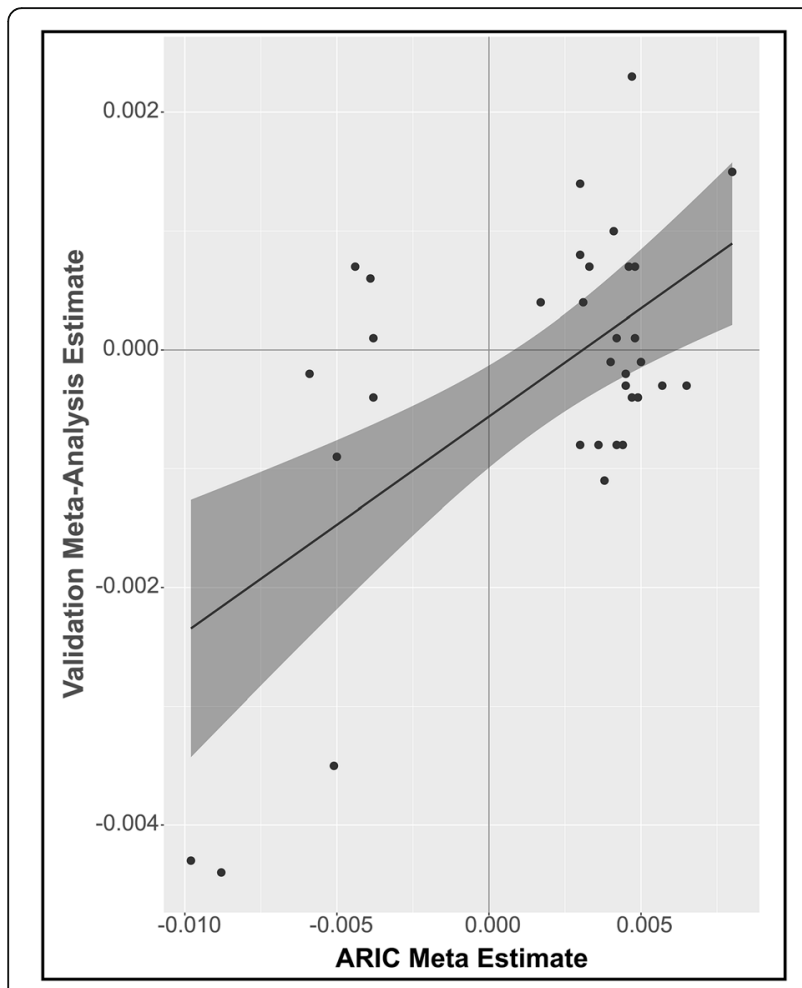

Fig. 2 Validation of meta-analysis identified CpGs in CHS and FHS combined cohorts ( $N=2528, R^{2}=0.36$, Kendall tau $\left.=0.19\right)$

systems, we note that three validated mtDNA-CNassociated CpGs showed nominally significant differential expression $(P<0.05)$ and two were significant after Bonferroni correction $(P<0.01$,Additional file 2 : Table S7, Additional file 1: Fig. S8). We also observe no difference in global CpG methylation patterns (across all CpGs sites interrogated) between negative control and TFAM knockout cell lines (Additional file 1: Fig. S9). RNA-seq resulted in clustering of knockout and control lines (Additional file 1: Fig. S10). All nominally differentially expressed genes $(P<0.05)$ within $1 \mathrm{Mb}$ of the TFAM knockout differentially methylated $\mathrm{CpGs}$ were identified (Additional file 2: Table S8). Five genes nearby the three differentially methylated CpGs were differentially expressed after Bonferroni correction for the number of genes within $1 \mathrm{Mb}$ of each CpG $\left(P<6.41 \times 10^{-4}\right)$ (Table 2). The five differentially expressed genes were as follows: IFI35 $\left(P=3.76 \times 10^{-5}\right)$ and RAMP2 $\left(P=5.51 \times 10^{-4}\right)$ near cg26094004; RPIA near $\operatorname{cg} 26563141(P=5.04 \times$ $\left.10^{-6}\right)$; and HLA-DRB5 $\left(P=6.50 \times 10^{-7}\right)$ and MSH5 $\left(P=2.50 \times 10^{-4}\right)$ near cg08899667.

Establishing causality via Mendelian randomization (MR): nuclear DNA methylation does not appear to be causative of changes in mtDNA-CN at identified CpGS Mendelian randomization (MR), a form of instrument variable analysis, is a well-established method for the assessment of causal relationships. Specifically, MR seeks to establish causality by exploiting the fact that SNPs are assigned at conception and randomly distributed in the population making them an excellent instrument variable to determine the relationship between modifiable exposures and relevant outcomes. We used MR to further test the direction of causality between mtDNA-CN (outcome) and nuclear methylation (exposure) by exploring the relationship between

\section{ESTABLISHING CAUSATION}

mtDNA-CN $\rightarrow$ nuclear methylation

TFAM KNOCKOUT

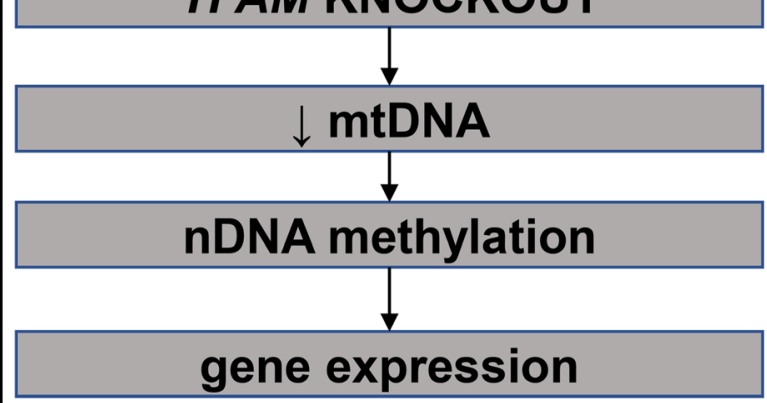

nuclear methylation $\rightarrow$ mtDNA-CN

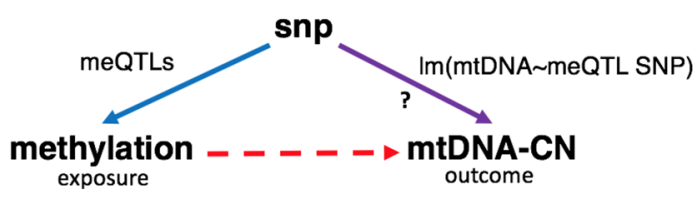

Mendelian randomization with methylation as the exposure and mtDNA-CN as the outcome

Fig. 3 Methods used to establish causation 


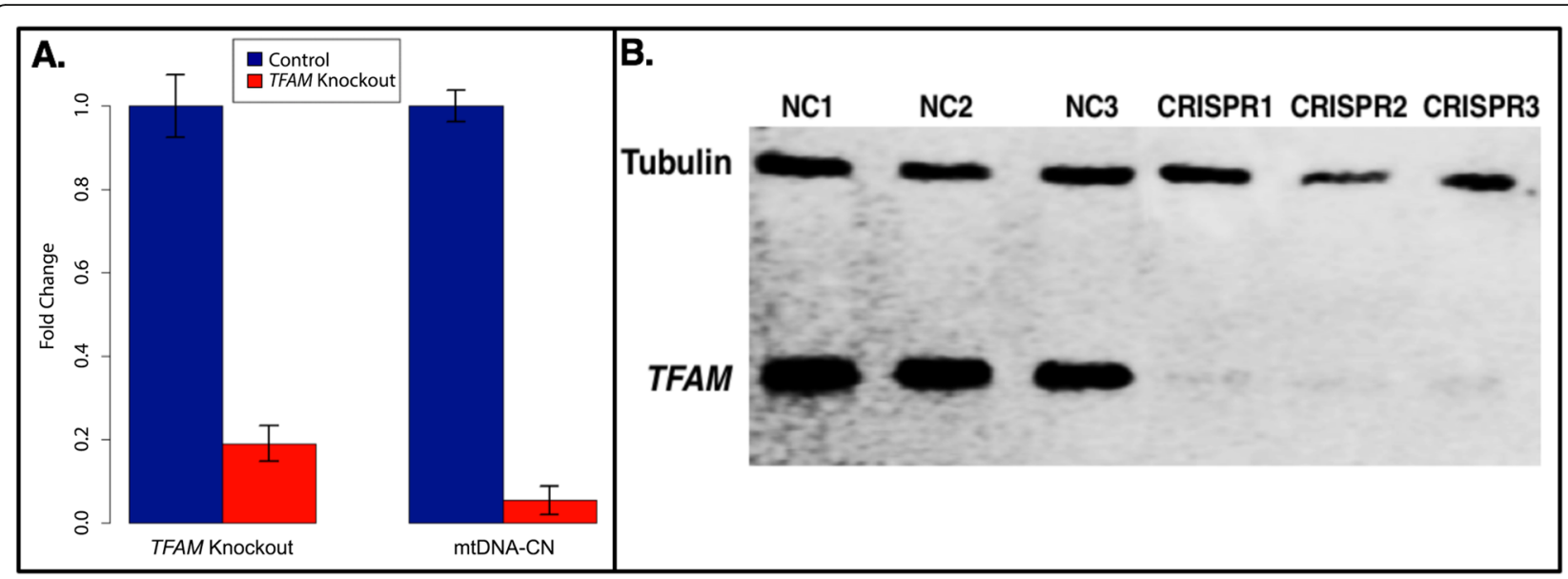

Fig. 4 CRISPR-Cas9-induced heterozygous knockout of TFAM reduced RNA expression, mtDNA-CN, and protein levels. a RNA expression was reduced by over $80 \%$ relative to negative control (NC) expression (left) (passage 45 ). mtDNA-CN levels showed an 18-fold reduction in TFAM knockout cell lines, (passage 32) (right). b Western blot of CRISPR TFAM heterozygous knockout showed a significant reduction (> 81\%) in TFAM protein (passage 35). NC = negative control lines. CRISPR = CRISPR TFAM knockout lines. Control is Tubulin

methylation quantitative trait loci (meQTLs) (instrument variable) and mtDNA-CN (Fig. 3, Additional file 2: Table S9). Specifically, if nDNA methylation at our sites of interest is causative of changes in mtDNA$\mathrm{CN}$, then meQTL single nucleotide polymorphisms (SNPs) for these CpGs of interest would be expected to also be associated with mtDNA-CN. Alternatively, if mtDNA-CN is not associated with meQTL SNPs, then it would follow that changes to nDNA methylation likely do not drive changes to mtDNA-CN at these CpGs, or suggest that other factors are modulating these interactions.

We identified four independent cis meQTLs in the ARIC EA cohort (permuted $P=7.84 \times 10^{-4}$ ) and six independent cis meQTLs in the ARIC AA cohort (permuted $P=9.12 \times 10^{-4}$ ) across five mtDNA-CN associated CpGs for use as an instrument variable for MR (Additional file 2: Table S9A). We further identified two independent discovery meta-analysis-derived meQTLs by combining results from ARIC EA and AA cohorts (permuted $P=3.97 \times 10^{-5}$, fixed effects (FE) model) (Additional file 2: Table S9B).

We then assessed the relationship between meQTL SNPs and mtDNA-CN. The results of the MR were null for each independent meQTL (Bonferroni $P=0.005$ ) (Additional file 2: Table S9). While our power for a single meQTL varied depending on the specific meQTL assessed, with power to detect an individual association ranging from 0.18 to 0.99 across the 12 meQTLs, overall power was $>99 \%$ to detect at least one associated meQTL. These results support the experimentally established direction of causality by suggesting that modification of $\mathrm{nDNA}$ methylation at $\mathrm{CpG}$ sites of interest does not drive alterations in mtDNA-CN.

\section{Association of $\mathrm{CpG}$ methylation with mtDNA-CN- associated phenotypes}

Since decreased mtDNA-CN has been associated with a number of aging-related diseases, and given our hypothesis that mtDNA-CN leads to nDNA methylation changes which influence disease outcomes, associated CpGs should also be associated with mtDNA-CNrelated phenotypes. To test these associations, we performed logistic regression and survival analysis for prevalent and incident diseases, respectively, for each of the six validated $\mathrm{CpGs}$ as they relate to coronary heart disease (CHD), cardiovascular disease (CVD), and mortality in the ARIC, FHS, and CHS cohorts (Table 3, Additional file 7: Table S10). Results from each cohort were meta-analyzed to derive an overall association for each validated $\mathrm{CpG}$ with each outcome of interest.

We identify nominally significant phenotype associations with at least one of the mtDNA-CN-associated traits of interest for four of the six validated mtDNA$\mathrm{CN}$-associated CpGs $(P<0.05)$. Specifically, results in the expected direction of effect for prevalent $\mathrm{CHD}$ and prevalent CVD were identified for two mtDNACN associated CpGs (cg26094004 and cg08899667). Similarly, results in the expected direction of effect were identified for the association between all-cause mortality and cg26563141 and cg08899667. Thus, we found cg08899667 to be nominally associated with three of the five mtDNA-CN-associated phenotypes, including all-cause mortality (Table 3).

The association between mtDNA-CN and each related phenotype does not change considerably with and without inclusion of mtDNA-CN-associated $\mathrm{CpGs}$ in the model (Fig. 5). This likely reflects mtDNA-CN acting on multiple biological pathways and the requirement for an 


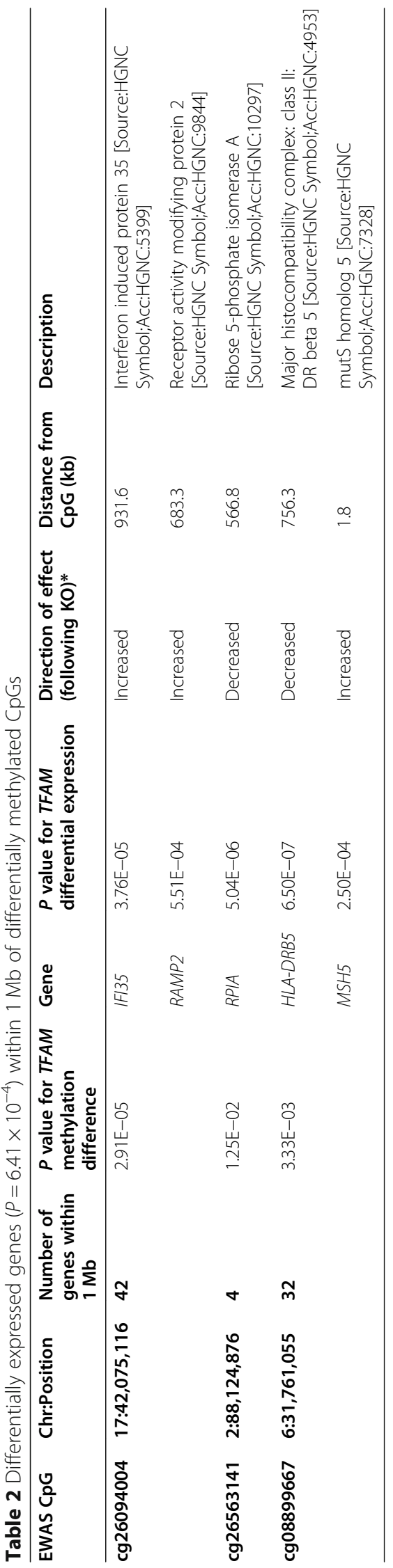


Table 3 Summary of phenotype associations from all-cohort meta-analysis for Validated CpGs. Bold entries highlight nominally significant associations $(P<0.05)$. *Significant after stringent multiple test correction

\begin{tabular}{|c|c|c|c|c|c|c|c|c|c|c|c|c|c|c|c|c|c|}
\hline \multirow[t]{2}{*}{ All cohorts } & \multirow{2}{*}{$\begin{array}{l}\text { Meta } \\
\text { direction }\end{array}$} & \multirow{2}{*}{$\begin{array}{l}\text { Expected } \\
\text { direction } \\
\text { of } \\
\text { phenotype } \\
\text { association }\end{array}$} & \multicolumn{3}{|c|}{ Prevalent CHD } & \multicolumn{3}{|c|}{ Prevalent CVD } & \multicolumn{3}{|c|}{ Incident CHD } & \multicolumn{3}{|c|}{ Incident CVD } & \multicolumn{3}{|c|}{ Mortality } \\
\hline & & & Beta & $\begin{array}{l}\text { Std. } \\
\text { Error }\end{array}$ & $\begin{array}{l}P \\
\text { value }\end{array}$ & Beta & $\begin{array}{l}\text { Std. } \\
\text { Error }\end{array}$ & $\begin{array}{l}P \\
\text { value }\end{array}$ & Beta & $\begin{array}{l}\text { Std. } \\
\text { Error }\end{array}$ & $\begin{array}{l}P \\
\text { value }\end{array}$ & Beta & $\begin{array}{l}\text { Std. } \\
\text { Error }\end{array}$ & $\begin{array}{l}P \\
\text { value }\end{array}$ & Beta & $\begin{array}{l}\text { Std. } \\
\text { Error }\end{array}$ & $\begin{array}{l}P \\
\text { value }\end{array}$ \\
\hline $\operatorname{cg} 21051031$ & Positive & Negative & -0.56 & 1.95 & $\begin{array}{l}7.74 \mathrm{E} \\
-01\end{array}$ & -0.67 & 1.88 & $\begin{array}{l}7.23 \mathrm{E} \\
-01\end{array}$ & 0.96 & 1.80 & $\begin{array}{l}5.94 \mathrm{E} \\
-01\end{array}$ & -0.28 & 1.41 & $\begin{array}{l}8.43 \mathrm{E} \\
-01\end{array}$ & -0.79 & 0.81 & $\begin{array}{l}3.31 \mathrm{E} \\
-01\end{array}$ \\
\hline cg26094004 & Negative & Positive & 2.91 & 1.48 & $\begin{array}{l}4.89 \mathrm{E} \\
-02\end{array}$ & 3.13 & 1.40 & $\begin{array}{l}2.55 \mathrm{E} \\
-02\end{array}$ & 0.89 & 0.93 & $\begin{array}{l}3.39 \mathrm{E} \\
-01\end{array}$ & -0.13 & 0.77 & $\begin{array}{l}8.63 \mathrm{E} \\
-01\end{array}$ & -0.78 & 0.49 & $\begin{array}{l}1.16 \mathrm{E} \\
-01\end{array}$ \\
\hline $\operatorname{cg} 26563141$ & Negative & Positive & 0.22 & 1.33 & $\begin{array}{l}8.69 \mathrm{E} \\
-01\end{array}$ & -0.31 & 1.24 & $\begin{array}{l}8.00 \mathrm{E} \\
-01\end{array}$ & 0.13 & 0.94 & $\begin{array}{l}8.88 \mathrm{E} \\
-01\end{array}$ & 1.10 & 0.75 & $\begin{array}{l}1.40 \mathrm{E} \\
-01\end{array}$ & 0.98 & 0.45 & $\begin{array}{l}2.87 \mathrm{E} \\
-02\end{array}$ \\
\hline cg14575356 & Positive & Negative & -1.80 & 2.04 & $\begin{array}{l}3.79 \mathrm{E} \\
-01\end{array}$ & -2.19 & 1.88 & $\begin{array}{l}2.45 \mathrm{E} \\
-01\end{array}$ & 2.71 & 1.34 & $\begin{array}{l}4.36 \mathrm{E} \\
-02\end{array}$ & 1.75 & 1.08 & $\begin{array}{l}1.04 \mathrm{E} \\
-01\end{array}$ & 0.12 & 0.68 & $\begin{array}{l}8.63 \mathrm{E} \\
-01\end{array}$ \\
\hline $\operatorname{cg} 23513930$ & Positive & Negative & 1.99 & 3.37 & $\begin{array}{l}5.54 \mathrm{E} \\
-01\end{array}$ & 0.88 & 3.07 & $\begin{array}{l}7.75 \mathrm{E} \\
-01\end{array}$ & 2.24 & 1.84 & $\begin{array}{l}2.24 \mathrm{E} \\
-01\end{array}$ & 0.50 & 1.49 & $\begin{array}{l}7.39 \mathrm{E} \\
-01\end{array}$ & 1.07 & 0.94 & $\begin{array}{l}2.53 \mathrm{E} \\
-01\end{array}$ \\
\hline cg08899667 & Negative & Positive & 3.65 & 1.76 & $\begin{array}{l}3.81 \mathrm{E} \\
-02\end{array}$ & 3.35 & 1.61 & $\begin{array}{l}3.81 \mathrm{E} \\
-02\end{array}$ & 1.57 & 1.10 & $\begin{array}{l}1.54 \mathrm{E} \\
-01\end{array}$ & 0.06 & 0.93 & $\begin{array}{l}9.44 \mathrm{E} \\
-01\end{array}$ & 2.08 & 0.59 & $\begin{array}{l}3.93 \mathrm{E} \\
-04^{*}\end{array}$ \\
\hline
\end{tabular}

accumulation of many methylation changes at multiple CpGs to impact disease outcomes.

\section{Discussion}

We report evidence that changes in mtDNA-CN influence nDNA methylation at specific, validated loci, including those acting in the "neuroactive ligand receptor interaction" pathway which may impact human health and disease via altered cell signaling. A number of these associations were validated across two independent cohorts and identified both cross-sectionally and experimentally. It is important to note that the methods used to estimate mtDNA-CN differed between the three cohorts with a qPCR-based approach used for CHS, a whole genome sequencing approach for FHS and microarray analysis for ARIC. This may reflect the robustness of results across mtDNA-CN estimation methods and also explain why some but not all CpGs replicated in our validation analysis [55]. We also note that as expected, our experimental approach using kidney cell

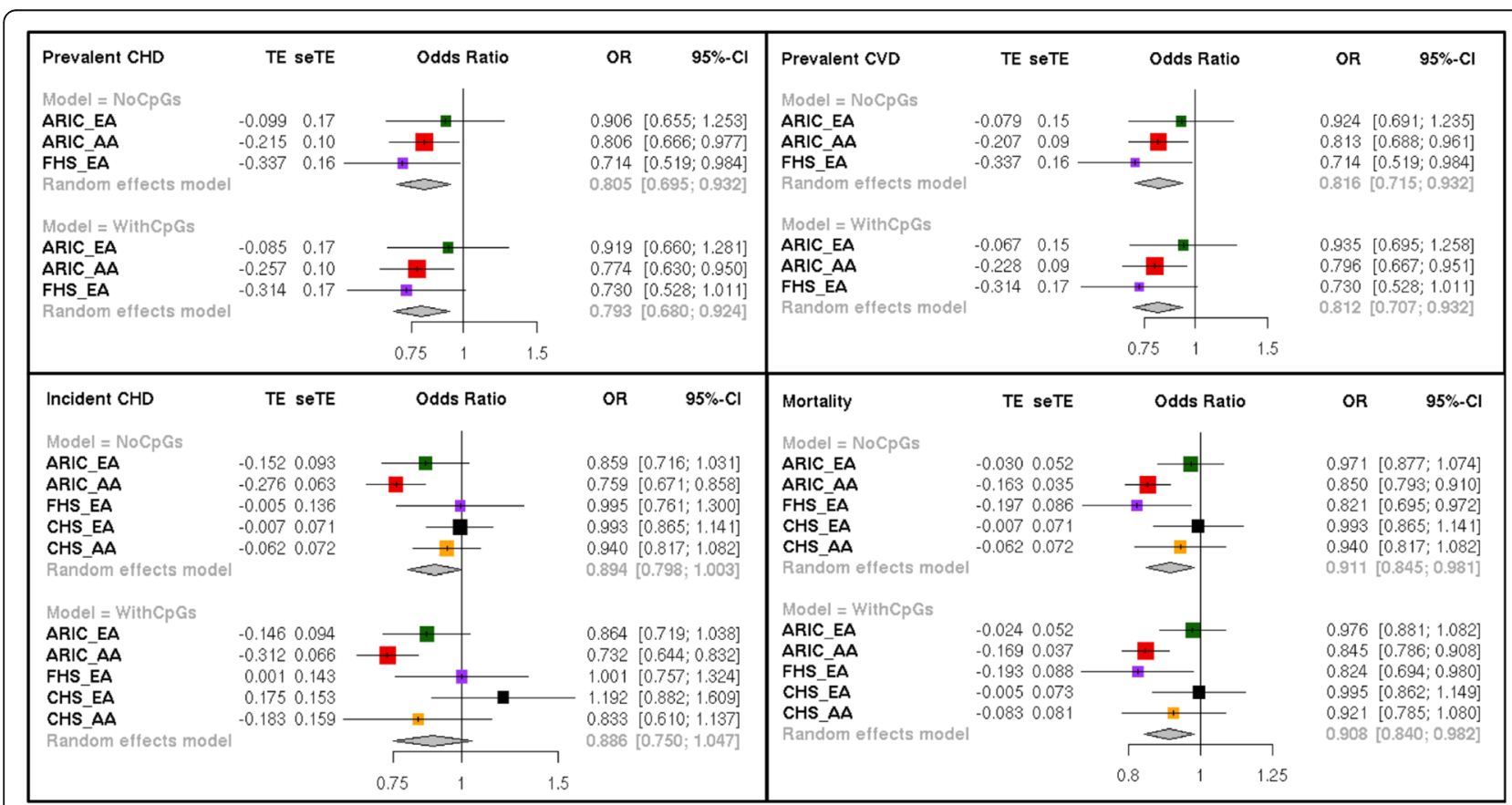

Fig. 5 Cohort-specific phenotype associations with mtDNA-CN. All models were adjusted for age, sex, center, genotyping PCs, and where indicated for mtDNA-CN-associated CpGs 
lines replicated some but not all of the pathway-level results from blood. These findings likely reflect both the intrinsic differences between cell line data and crosssectional data as well as the inherent complexity of mitochondrial-to-nuclear signaling which would be expected to vary across cell types, developmental timepoints, and environmental conditions.

\section{DNA methylation as a link between mtDNA-CN and changes in nuclear gene expression}

The relationship between the nuclear and mitochondrial genomes strongly implicates communication between them as vital for proper cell functioning. Given the function of the mitochondria in meeting cellular energy demands, mitochondria may play an important role in translating environmental stimuli into epigenetic changes. Accordingly, mtDNA-CN levels are sensitive to a number of chemicals [56], highlighting the role of mtDNA as an environmental biosensor. We hypothesize that modification of nDNA methylation via mitochondrial signaling modifies gene expression which in turn may lead to disease outcomes or influence severity of disease. Supporting this, epigenetic changes in nuclear DNA correlate with reduced cancer survival and low mtDNA-CN correlates with poor survival across a number of cancer types $[57,58]$. Thus, retrograde signals from the mitochondria to the nucleus may be crucial in sensing homeostasis and translating extracellular signals into altered gene expression [18].

Our results implicate the neuroactive ligand receptor interaction pathway and in general additional processes involved in cellular signaling. The results also show that although the same pathways are implicated across our independent datasets, the specific genes affected differ between conditions. Interestingly, the neuroactive ligand receptor interaction pathway has been identified as having the second highest number of atherosclerosis candidate genes of any KEGG pathway, harboring 53 atherosclerosis candidate genes (272 total genes in the pathway) [57-59]. This is an interesting finding given the association of mtDNA-CN with cardiovascular disease [6-8]. Perhaps unsurprisingly, this pathway also belongs to the class of KEGG pathways that are responsible for environmental information processing and signaling molecules/interactions. Genes from this pathway are highly expressed across a wide variety of tissues including whole blood, heart tissue, and cardia myocytes, among others (http://software.broadinstitute. org/gsea/). We further observe synaptic signaling to be significantly associated, which is supported by observations that synaptic signals can induce changes in nDNA methylation leading to plasticity-related gene expression changes [60].

\section{Proposed mechanisms for the methylation of nDNA as a result of changes in mtDNA}

The precise identity of the signal(s) coming from the mitochondria that might be responsible for modifying nDNA methylation has not yet been identified and warrants further experimentation. It is however possible that histones may play a role in this signaling process. Supporting this, mitochondria-to-nucleus retrograde signaling has been shown to regulate histone acetylation and alter nuclear gene expression through the heterogenous ribonucleoprotein A2 (hnRNAP2, 25]. In fact, histone modifications co-vary with mitochondrial content and are linked with chromatin activation, namely H4K16, H3L4me3, and H3K36me2 [61]. In addition, perturbations to oxidative phosphorylation alter methylation processes by modifications to the methionine cycle. Methionine metabolism is essential for production of $S$-adenosylmethioninine (SAM), a methyl donor for histone and DNA methyltransferases [62]. Specifically, three DNA methyltransferases (DNMTs), DNMT1, DNMT3a, and DNMT3b, mediate methyl transfer from SAM to cytosine whereby SAM is the electrophilic methyl source to produce 5-methylcytosine at CpG sites in double-stranded DNA [63]. Alpha-ketoglutarate, a krebs cycle intermediate, is also involved in cytosine demethylation as a cofactor for the Tet family of dioxygenases via oxidation of 5methylcytosine to 5-hydroxymethylcytosine by flipping the target cytosine out and into the active site [64]. Further experimentation to determine the biological mechanisms at play is essential.

Uncovering the precise nature of this signaling from mitochondria to the nucleus would be expected to expose essential clues that will integrate epigenetic regulation, mitochondrial and genomic polymorphisms, and complex phenotypes. Further assessment of the functional mechanisms underlying the crosstalk between mtDNA-CN, methylation, and disease through sophisticated cell culture models will be required to fully appreciate the diagnostic and therapeutic utility of the interaction between mtDNA and nDNA as identified in this study.

\section{Influence of findings on complex disease etiology}

The observation that differential methylation occurred at specific-sites throughout the nuclear genome as a result of changes to mtDNA-CN, provides an explanation for how mtDNA could alter normal homeostasis as well as susceptibility and/or severity of diseases. The association of mtDNA-CN-associated CpGs with mtDNA-CNrelated disease states lends further support to the hypothesis that modulation of mtDNA-CN not only modifies the nuclear epigenome, and the expression of nearby genes, but does so at locations which may be relevant to disease outcomes, including cardiovascular disease and 
all-cause mortality. In particular, these observations may explain how mitochondrial-to-nuclear signaling could influence polygenic traits with complex etiology and in particular those for which environmental insults play a role. However, mtDNA-CN is likely acting through a number of biological pathways to impact disease and thus mtDNA-CN-associated CpGs would be expected to account for a subset of the effect of mtDNA-CN on disease.

Further, these findings have direct implications for the recent emergence of mitochondrial donation in humans as they suggest that mitochondrial replacement into recipient oocytes may lead to unexpected changes to the nuclear epigenome. Thus, unraveling the complex interplay of the mitochondria and nucleus is also critical to properly informing medical decision makers.

This study design had a number of strengths and limitations. A possible limitation of the cross-sectional analysis is the potential for some common factor we have not been able to account for to influence both mtDNA$\mathrm{CN}$ and nDNA methylation. We also note that future analyses in larger admixed cohorts can identify and validate race-specific loci of interest. In experimental analysis, we used HEK293T cells for our knockdown studies due to the optimized protocols available as well as their propensity for transfection; however, we note that the use of a blood cell line may be more relevant to direct interpretation of the results. Further, the TFAM knockout reduced mtDNA-CN to levels which represent a greater reduction than that seen in population samples reflecting a more drastic change than is likely to be caused by natural variation alone. We also note that the TFAM knockout may independently influence nDNA methylation though unknown mechanisms that we did not account for in this study and/or TFAM may have an effect on differentially expressed genes that is independent of the epigenome. Additionally, given that transcriptional activation/repression can be regulated over large distances in the genome, our approach using the nearest gene for pathway analysis may have limited the number of associations identified. Further, prevalent disease is subject to reverse causality and therefore the results on prevalent phenotypes should be interpreted with caution. Strengths of this study include the well phenotyped and carefully collected incident disease data, the robustness of the findings across multiple cohorts and ethnic groups, and the careful quality control employed. Further, our results stood up to rigorous permutation testing which increases the reliability of these observations.

\section{Conclusions}

Cross-sectionally, we have shown that variation in mtDNA-CN is associated with nuclear epigenetic modifications at specific CpGs across multiple independent cohorts. Specifically, six mtDNA-CN-associated CpGs were robustly identified across three independent cohorts. Second, we found meQTL SNPs to not be associated with mtDNA-CN, suggesting that nuclear methylation at these $\mathrm{CpGs}$ does not result in alterations to mtDNA-CN. Third, functional results show that modulation of mtDNA-CN leads to differential methylation and expression of genes relating to cell signaling processes. Further, mtDNA-CN-associated CpGs display association with mtDNA-CN-related phenotypes, namely cardiovascular disease and all-cause mortality. These findings demonstrate that the mechanism(s) by which mtDNA-CN influences disease is at least in part via regulation of nuclear gene expression through modification of nDNA methylation. Specifically, the data presented here support the model that modification of mtDNA-CN leads to changes to nDNA methylation which in turn influence nuclear DNA expression of nearby genes which contribute to disease pathology. These results have implications for understanding the mechanisms behind mitochondrial and nuclear communication as it relates to complex disease etiology as well as the consequences of mitochondrial replacement therapeutic strategies. Taken together, the results confirm that in elucidating the underpinnings of complex disease, knowledge of only nuclear DNA dynamics is not sufficient to fully elucidating disease etiology.

\section{Supplementary information}

Supplementary information accompanies this paper at https://doi.org/10. 1186/s13073-020-00778-7.

\begin{abstract}
Additional file 1: Supplementary Methods and Supplemental Figures: Fig. S1. QQ plots from ARIC association analyses. Fig. S2. Manhattan plot of association between nuclear DNA methylation and mtDNA-CN in ARIC (discovery cohort) stratified analysis. Fig. S3. Correlation between beta estimates for significant CpGs identified by ARIC EA and ARIC AA analyses (Discovery cohort). Fig. S4. Volcano Plots of results from ARIC association analyses. Fig. S5. Cohort correlations between beta estimates for genome-wide significant CpGs in discovery cohort (ARIC) and beta estimates from CHS/FHS validation cohorts. Fig. S6. Individual cohort results for 6 validated CpGs. Fig. S7. qPCR DNA Copy Number results for TFAM gene in negative control and TFAM knockout lines. Fig. S8. ARIC identified CpGs with methylation differences between EPIC Negative Control (NC) lines and TFAM knockout (CRISPR) cell lines. Fig. S9. Global methylation patterns in negative Control (NC) and CRISPR-TFAM knockout (CR) cell lines. Fig. S10. TFAM knockout RNA sequencing clustering heatmap of TFAM knockout and control cell lines.
\end{abstract}

Additional file 2: Table S1. Sample characteristics of discovery and validation cohorts. Table S6. Neuroactive-ligand receptor interaction genes as identified by KEGG analysis in each approach, number of genes used in each analysis and neuroactive ligand enrichment $P$-value are included in brackets. Table S7. Methylation Status of Validated CpGs in TFAM KO cell lines $(N=6)$. Bolded entries indicate differential expression $P<0.05$. Table S8. Differentially expressed genes $(P<0.05)$ within $1 \mathrm{Mb}$ of differentially methylated CpGs in TFAM knockout cell lines. Shading indicates most differentially expressed gene for each CpG. Table S9. Results of Mendelian Randomization. A. Results for association between ARIC EA and AA derived independent cis meQTLs and mtDNA-CN. B. Results for 
association between ARIC meta-analysis derived independent cis meQTLs and mtDNA-CN (fixed effects model).

Additional file 3: Table S2. ARIC summary statistics. A. Meta-Analysis, B. AA, C. EA.

Additional file 4: Table S3. A. Results for 34 independent ARIC Discovery Meta-Analysis identified mtDNA-CN associated CpGs across all studied cohorts and Validation Meta-Analysis/All Cohort Meta-Analysis. Validation meta-analysis included CHS AA, CHS EA and FHS EA cohorts ( $P<0.05$ and same direction, bolded cells). All cohort meta-analysis (ARIC AA, ARIC EA, CHS AA, CHS EA and FHS EA) identified 6 validated CpGs $(P<5 \times 10-8$, shaded cells). B. Results for 23 independent ARIC AA identified CpGs across all studied cohorts. C. Results for 15 independent ARIC EA identified CpGs across all studied cohorts.

Additional file 5: Table_S4. TFAM Methylation analysis summary statistics.

Additional file 6: Table_S5.TFAM Expression analysis summary statistics. Additional file 7: Table_S10. Phenotype analysis: Cohort specific results and meta-analysis of all cohorts.

\section{Abbreviations}

AA: African American; ARIC: Atherosclerosis Risk in Communities; CHD: Coronary heart disease; CHS: Cardiovascular Health Study; CVD: Cardiovascular disease; EA: European American; FHS: Framingham Heart Study; meQTLs: Methylation quantitative trait loci; MR: Mendelian randomization; mtDNA: Mitochondrial DNA; mtDNA-CN: Mitochondrial DNA copy number; nDNA: Nuclear DNA; qPCR: Quantitative polymerase chain reaction; SNPs: Single-nucleotide polymorphisms; SVA: Surrogate variable analysis; TOPMed: Trans-Omics in Precision Medicine; WGS: Whole genome sequencing

\section{Acknowledgements}

Infinium Methylation EPIC BeadChip array hybridization was performed at the UTHealth Human Genetics Center, The University of Texas, Houston, TX Illumina sequencing was conducted at the Genetic Resources Core Facility, Johns Hopkins Institute of Genetic Medicine, Baltimore, MD.

\section{ARIC Acknowledgements}

The Atherosclerosis Risk in Communities study has been funded in whole or in part with Federal funds from the National Heart, Lung, and Blood Institute, National Institutes of Health, Department of Health and Human Services (contract numbers HHSN268201700001I, HHSN268201700002I, HHSN268201700003I, HHSN268201700004I and HHSN268201700005I). The authors thank the staff and participants of the ARIC study for their important contributions. Funding was also supported by 5RC2HL102419 and R01NS087541.

\section{CHS Acknowledgements}

Infrastructure for the CHARGE Consortium is supported in part by the National Heart, Lung, and Blood Institute grant R01HL105756. The CHS research was supported by NHLBI contracts HHSN268201200036C, HHSN268200800007C, HHSN268201800001C, N01HC55222, N01HC85079, N01HC85080, N01HC85081, N01HC85082, N01HC85083, N01HC85086; and NHLBI grants U01HL080295, U01HL130114, K08HL116640, R01HL087652, R01HL092111, R01HL103612, R01HL103612, R01HL111089, R01HL116747, and R01HL120393 with additional contribution from the National Institute of Neurological Disorders and Stroke (NINDS). Additional support was provided through R01AG023629 from the National Institute on Aging (NIA), Merck Foundation / Society of Epidemiologic Research as well as Laughlin Family, Alpha Phi Foundation, and Locke Charitable Foundation. A full list of principal CHS investigators and institutions can be found at CHS-NHLBI.org. The provision of genotyping data was supported in part by the National Center for Advancing Translational Sciences, CTSI grant UL1TR000124, and the National Institute of Diabetes and Digestive and Kidney Disease Diabetes Research Center (DRC) grant DK063491 to the Southern California Diabetes Endocrinology Research Center. The content is solely the responsibility of the authors and does not necessarily represent the official views of the National Institutes of Health.

\section{FHS Acknowledgements}

Whole genome sequencing (WGS) for the Trans-Omics in Precision Medicine (TOPMed) program was supported by the National Heart, Lung and Blood In stitute (NHLBI). WGS for "NHLBI TOPMed: Whole Genome Sequencing and Related Phenotypes in the Framingham Heart Study" (phs000974.v1.p1) was performed at the Broad Institute of MIT and Harvard (3R01HL092577-06S1, 3U54HG003067-12S2). Centralized read mapping and genotype calling, along with variant quality metrics and filtering, were provided by the TOPMed Informatics Research Center (3R01HL-117626-02S1, contract HHSN268201800002I). Phenotype harmonization, data management, sampleidentity QC, and general study coordination were provided by the TOPMed Data Coordinating Center (R01HL-120393; U01HL-120393; contract HHSN268201800001I). We gratefully acknowledge the studies and participants who provided biological samples and data for TOPMed.

This work is supported by National Institutes of Health $(\mathrm{NIH})$ contract N01HC-25195 and HHSN268201500001I and grant R01 HL092577, also supported by intramural funding of Dan Levy, National Heart, Lung, and Blood Institute (NHLBI) (for DNA methylation profiling), and Trans-Omics for Precision Medicine (TOPMed) sponsored by NHLBI/NIH. The Framingham Heart Study thanks the study participants and the multitude of investigators who over its 70-year history continue to contribute so much to further our knowledge of heart, lung, blood, and sleep disorders and associated traits.

The views expressed in this manuscript are those of the authors and do not necessarily represent the views of the National Heart, Lung, and Blood Institute; the National Institutes of Health; or the U.S. Department of Health and Human Services.

\section{Authors' contributions}

C.A.C., E.G., N.P., B.O., J.C., and D.E.A. conceived and designed the experiments. C.A.C., R.J.L., C.E.N., J.A.S., J.A.L., J.A.B., T.M.B., M.L.G., M.F., J.S.F., J.B. J.S.P, A.T., B.O., E.G., N.P., K.D.T., P.W., C.L., E.B., and D.E.A. acquired, analyzed, and interpreted the data. C.A.C. and D.E.A. were responsible for the drafting of the manuscript. C.A.C., R.J.L., J.S.F., C.L., A.T., M.F., B.O., J.A.B., J.S.P, T.M.B., and D.E.A. critically revised the manuscript for important intellectual content. C.A.C., R.J.L., J.A.L., J.A.B., C.L., E.G., N.P., and D.E.A. performed statistical analysis. J.C., E.G., E.B., and D.E.A. obtained funding. C.E.N., J.A.S., M.L.G., and J.B. provided administrative, technical, or material support. This work was performed under the supervision of N.S., D.L., E.G., and D.E.A. All authors read and approved the final manuscript.

\section{Funding}

This research was supported by grant R01HL131573 from the US National Institutes of Health. Castellani was supported by a CIHR Postdoctoral Fellowship. Cohort funding information can be found in the cohort-specific acknowledgement sections.

\section{Availability of data and materials}

The cell line datasets (methylation and gene expression) generated and analyzed during the current study are available in the NCBI Gene Expression Omnibus repository (GEO; http://www.ncbi.nlm.nih.gov/geo/) under accession numbers GSE133994 [44] and GSE134048 [46].

\section{Ethics approval and consent to participate}

The Atherosclerosis Risk in Communities (ARIC) study, Cardiovascular Health Study (CHS), and Framingham Heart Study (FHS) have been approved by the Institutional Review Board (IRB) at each participating institution. All participants provided written informed consent.

The ARIC study design and methods were approved by four different IRBs at each of the collaborating medical institutions: University of Mississippi Medical Center Institutional Review Board (Jackson Field Center); Wake Forest University Health Sciences Institutional Review Board (Forsyth County Field Center); University of Minnesota Institutional Review Board (Minnesota Field Center); and Johns Hopkins University School of Public Health Institutional Review Board (Washington County Field Center)

FHS is approved by the IRB at Boston University Medical Center. CHS recruited participants from Medicare lists at four sites and IRBs at each site were involved in human subject approval.

This research conforms to the principles of the Helsinki Declaration.

\section{Consent for publication}

Not applicable. 


\section{Competing interests}

The authors declare that they have no competing interests.

\begin{abstract}
Author details
${ }^{1}$ McKusick-Nathans Department of Genetic Medicine, Johns Hopkins University School of Medicine, Baltimore, MD, USA. ${ }^{2}$ Department of Laboratory Medicine and Pathology, University of Minnesota School of Medicine, Minneapolis, MN, USA. ${ }^{3}$ Human Genetics Center, School of Public Health, University of Texas Health Science Center at Houston, Houston, TX, USA. ${ }^{4}$ Cardiovascular Health Research Unit, Department of Medicine, University of Washington, Seattle, WA, USA. ${ }^{5}$ Department of Biostatistics, University of Washington, Seattle, WA, USA. Institute for Translational Genomics and Population Sciences, Los Angeles BioMedical Research Institute at Harbor-UCLA Medical Center, Torrance, CA, USA. ${ }^{7}$ Framingham Heart Study, Framingham, MA, USA. ${ }^{8}$ Population Sciences Branch, National Heart, Lung, and Blood Institute, National Institutes of Health, Bethesda, MD, USA. ${ }^{9}$ Department of Epidemiology and the Welch Center for Prevention, Epidemiology and Clinical Research, Johns Hopkins Bloomberg School of Public Health, Baltimore, MD, USA. ${ }^{10}$ Division of Epidemiology \& Community Health, School of Public Health, University of Minnesota, Minneapolis, MN, USA. " Brown Foundation Institute of Molecular Medicine, McGovern Medical School, University of Texas Health Science Center at Houston, Houston, TX, USA. ${ }^{12}$ Division of Cardiology, Department of Medicine, Johns Hopkins University School of Medicine, Baltimore, MD, USA. ${ }^{13}$ Department of Biostatistics, Boston University School of Public Health, Boston, MA, USA.
\end{abstract}

Received: 10 March 2020 Accepted: 4 September 2020

Published online: 28 September 2020

\section{References}

1. Clayton DA. Transcription and replication of animal mitochondrial DNAs. Int Rev Cytol. 1992;141:217-32

2. Pello R, Martín MA, Carelli V, Nijtmans LG, Achilli A, Pala M, et al. Mitochondrial DNA background modulates the assembly kinetics of OXPHOS complexes in a cellular model of mitochondrial disease. Hum Mol Genet. 2008;17:4001-11.

3. Wai T, Ao A, Zhang X, Cyr D, Dufort D, Shoubridge EA. The role of mitochondrial DNA copy number in mammalian fertility. Biol Reprod. 2010; 83:52-62.

4. Guha M, Avadhani NG. Mitochondrial retrograde signaling at the crossroads of tumor bioenergetics, genetics and epigenetics. Mitochondrion. 2013;13: 577-91.

5. Jeng J-Y, Yeh T-S, Lee J-W, Lin S-H, Fong T-H, Hsieh R-H. Maintenance of mitochondrial DNA copy number and expression are essential for preservation of mitochondrial function and cell growth. J Cell Biochem. 2008;103:347-57.

6. Dai D-F, Rabinovitch PS, Ungvari Z. Mitochondria and cardiovascular aging Circ Res. 2012;110:1109-24.

7. Ashar FN, Zhang Y, Longchamps RJ, Lane J, Moes A, Grove ML, et al. Association of mitochondrial DNA copy number with cardiovascular disease. JAMA Cardiol. 2017;2:1247-55.

8. Chen S, Xie X, Wang Y, Gao Y, Xie X, Yang J, et al. Association between leukocyte mitochondrial DNA content and risk of coronary heart disease: a case-control study. Atherosclerosis. 2014;237:220-6

9. Tin A, Grams ME, Ashar FN, Lane JA, Rosenberg AZ, Grove ML, et al. Association between mitochondrial DNA copy number in peripheral blood and incident CKD in the atherosclerosis risk in communities study. J Am Soc Nephrol. 2016;27:2467-73.

10. Ashar FN, Moes A, Moore AZ, Grove ML, Chaves PHM, Coresh J, et al. Association of mitochondrial DNA levels with frailty and all-cause mortality. J Mol Med. 2015;93:177-86.

11. Crovetto F, Lattuada D, Rossi G, Mangano S, Somigliana E, Bolis G, et al. A role for mitochondria in gestational diabetes mellitus? Gynecol Endocrinol. 2013;29:259-62.

12. Sookoian S, Rosselli MS, Gemma C, Burgueño AL, Fernández Gianotti T, Castaño GO, et al. Epigenetic regulation of insulin resistance in nonalcoholic fatty liver disease: impact of liver methylation of the peroxisome proliferatoractivated receptor y coactivator 1a promoter. Hepatology. 2010;52:1992-2000.

13. Pirola CJ, Scian R, Gianotti TF, Dopazo H, Rohr C, Martino JS, et al. Epigenetic modifications in the biology of nonalcoholic fatty liver disease: the role of DNA hydroxymethylation and TET proteins. Medicine (Baltimore) 2015;94:e1480

14. Delsite R, Kachhap S, Anbazhagan R, Gabrielson E, Singh KK. Nuclear genes involved in mitochondria-to-nucleus communication in breast cancer cells. Mol Cancer. 2002;1:6.

15. Kitamura E, Igarashi J, Morohashi A, Hida N, Oinuma T, Nemoto N, et al. Analysis of tissue-specific differentially methylated regions (TDMs) in humans. Genomics. 2007:89:326-37.

16. Horan MP, Cooper DN. The emergence of the mitochondrial genome as a partial regulator of nuclear function is providing new insights into the genetic mechanisms underlying age-related complex disease. Hum Genet 2014;133:435-58.

17. Cagin U, Enriquez JA. The complex crosstalk between mitochondria and the nucleus: what goes in between? Int J Biochem Cell Biol. 2015;63:10-5.

18. Vivian CJ, Brinker AE, Graw S, Koestler DC, Legendre C, Gooden GC, et al. Mitochondrial genomic backgrounds affect nuclear DNA methylation and gene expression. Cancer Res. 2017;77:6202-14.

19. Latorre-Pellicer A, Moreno-Loshuertos R, Lechuga-Vieco AV, Sánchez-Cabo F, Torroja C, Acín-Pérez R, et al. Mitochondrial and nuclear DNA matching shapes metabolism and healthy ageing. Nature. 2016;535:561-5.

20. Bellizzi D, D'Aquila P, Giordano M, Montesanto A, Passarino G. Global DNA methylation levels are modulated by mitochondrial DNA variants. Epigenomics. 2012;4:17-27.

21. Kelly RDW, Mahmud A, McKenzie M, Trounce IA, St John JC. Mitochondrial DNA copy number is regulated in a tissue specific manner by DNA methylation of the nuclear-encoded DNA polymerase gamma a. Nucleic Acids Res. 2012:40:10124-38.

22. Lee W, Johnson J, Gough DJ, Donoghue J, Cagnone GLM, Vaghjiani V, et al. Mitochondrial DNA copy number is regulated by DNA methylation and demethylation of POLGA in stem and cancer cells and their differentiated progeny. Cell Death Dis. 2015;6:e1664

23. Smiraglia DJ, Kulawiec M, Bistulfi GL, Gupta SG, Singh KK. A novel role for mitochondria in regulating epigenetic modification in the nucleus. Cancer Biol Ther. 2008;7:1182-90.

24. Xie C, Naito A, Mizumachi T, Evans TT, Douglas MG, Cooney CA, et al. Mitochondrial regulation of cancer associated nuclear DNA methylation. Biochem Biophys Res Commun. 2007;364:656-61.

25. Guha M, Srinivasan S, Guja K, Mejia E, Garcia-Diaz M, Johnson FB, et al. HnRNPA2 is a novel histone acetyltransferase that mediates mitochondrial stress-induced nuclear gene expression. Cell Discov. 2016;2:16045.

26. Sun X, Johnson J, St. John JC. Global DNA methylation synergistically regulates the nuclear and mitochondrial genomes in glioblastoma cells. Nucleic Acids Res 2018;46:5977-5995.

27. Sun X, St John JC. Modulation of mitochondrial DNA copy number in a model of glioblastoma induces changes to DNA methylation and gene expression of the nuclear genome in tumours. Epigenetics Chromatin. 2018 [cited 2020 May 13];11. Available from: https://www.ncbi.nlm.nih.gov/pmc/ articles/PMC6136172/.

28. The Atherosclerosis Risk in Communities (ARIC) Study: design and objectives. The ARIC investigators. Am J Epidemiol. 1989;129:687-702.

29. MitoPipeline G. MitoPipeline. MitoPipeline. 2018 [cited 2019 Nov 27]. Available from: http://genvisis.org/MitoPipeline/.

30. Leek JT, Johnson WE, Parker HS, Jaffe AE, Storey JD. The sva package for removing batch effects and other unwanted variation in high-throughput experiments. Bioinformatics. 2012;28:882-3.

31. Chen Y, Lemire M, Choufani S, Butcher DT, Grafodatskaya D, Zanke BW, et al. Discovery of cross-reactive probes and polymorphic CpGs in the Illumina Infinium HumanMethylation450 microarray. Epigenetics. 2013;8:203-9.

32. Teschendorff AE, Marabita F, Lechner M, Bartlett T, Tegner J, Gomez-Cabrero $D$, et al. A beta-mixture quantile normalization method for correcting probe design bias in Illumina Infinium 450 k DNA methylation data. Bioinformatics. 2013:29:189-96

33. Pidsley R, Wong CC, Volta M, Lunnon K, Mill J, Schalkwyk LC. A data-driven approach to preprocessing Illumina 450K methylation array data. BMC Genomics. 2013;14:293.

34. Fortin J-P, Labbe A, Lemire M, Zanke BW, Hudson TJ, Fertig EJ, et al. Functional normalization of 450k methylation array data improves replication in large cancer studies. Genome Biol. 2014 [cited 2018 Dec 16]; 15. Available from: https://www.ncbi.nlm.nih.gov/pmc/articles/PMC4283580/.

35. Houseman EA, Molitor J, Marsit CJ. Reference-free cell mixture adjustments in analysis of DNA methylation data. Bioinformatics. 2014;30:1431-9. 
36. Willer CJ, Li Y, Abecasis GR. METAL: fast and efficient meta-analysis of genomewide association scans. Bioinformatics. 2010;26:2190-1.

37. Fried LP, Borhani NO, Enright P, Furberg CD, Gardin JM, Kronmal RA, et al. The cardiovascular health study: design and rationale. Ann Epidemiol. 1991; 1:263-76.

38. Dawber TR, Meadors GF, Moore FE. Epidemiological approaches to heart disease: the Framingham study. Am J Public Health Nations Health. 1951;41: 279-81.

39. Ding J, Sidore C, Butler TJ, Wing MK, Qian Y, Meirelles O, et al. Assessing mitochondrial DNA variation and copy number in lymphocytes of $\sim 2,000$ Sardinians using tailored sequencing analysis tools. PLoS Genet. 2015;11: e1005306.

40. Roby J, Just Allan C, Marioni Riccardo E, Pilling Luke C, Reynolds Lindsay M, Mandaviya Pooja R, et al. Epigenetic signatures of cigarette smoking. Circ Cardiovasc Genet. 2016;9:436-47.

41. Shabalin AA. Matrix eQTL: ultra fast eQTL analysis via large matrix operations. Bioinformatics. 2012;28:1353-8.

42. Han B, Eskin E. Random-effects model aimed at discovering associations in meta-analysis of genome-wide association studies. Am J Hum Genet. 2011; 88:586-98.

43. Brion M-JA, Shakhbazov K, Visscher PM. Calculating statistical power in Mendelian randomization studies. Int J Epidemiol. 2013;42:1497-501.

44. Castellani C, Sumpter J, Newcomb C, Arking D. HEK293 TFAM Knockout Methylation Study. Gene Expression Omnibus (GEO); 2019. Available from: https://www.ncbi.nlm.nih.gov/geo/query/acc.cgi?acc=GSE133994.

45. Fortin J-P, Triche TJ, Hansen KD. Preprocessing, normalization and integration of the Illumina HumanMethylationEPIC array with minfi. Bioinformatics. 2017:33:558-60.

46. Castellani C, Sumpter J, Newcomb C, Arking D. HEK293 TFAM Knockout Expression Study. Gene Expression Omnibus (GEO); 2019. Available from: https://www.ncbi.nlm.nih.gov/geo/query/acc.cgi?acc=GSE134048.

47. Bray NL, Pimentel H, Melsted P, Pachter L. Near-optimal probabilistic RNAseq quantification. Nat Biotechnol. 2016;34:525-7.

48. Pimentel $\mathrm{H}$, Bray NL, Puente S, Melsted P, Pachter L. Differential analysis of RNAseq incorporating quantification uncertainty. Nat Methods. 2017;14:687-90.

49. Huang $\mathrm{H}$, Chanda P, Alonso A, Bader JS, Arking DE. Gene-based tests of association. PLoS Genet. 2011;7:e1002177.

50. Geeleher P, Hartnett L, Egan LJ, Golden A, Raja Ali RA, Seoighe C. Gene-set analysis is severely biased when applied to genome-wide methylation data. Bioinformatics. 2013;29:1851-7.

51. Young MD, Wakefield MJ, Smyth GK, Oshlack A. Gene ontology analysis for RNA-seq: accounting for selection bias. Genome Biol. 2010;11:R14.

52. Ekstrand MI, Falkenberg M, Rantanen A, Park CB, Gaspari M, Hultenby K, et al. Mitochondrial transcription factor a regulates mtDNA copy number in mammals. Hum Mol Genet. 2004;13:935-44.

53. de Oliveira VC, Moreira GSA, Bressan FF, Gomes Mariano Junior C, KCS R, Charpentier M, et al. Edition of TFAM gene by CRISPR/Cas9 technology in bovine model. PLoS ONE. 2019;14:e0213376.

54. Kanki T, Ohgaki K, Gaspari M, Gustafsson CM, Fukuoh A, Sasaki N, et al. Architectural role of mitochondrial transcription factor $A$ in maintenance of human mitochondrial DNA. Mol Cell Biol. 2004;24:9823-34.

55. Longchamps RJ, Castellani CA, Yang SY, Newcomb CE, Sumpter JA, Lane J, et al. Evaluation of mitochondrial DNA copy number estimation techniques. PLoS One. 2020;15:e0228166.

56. Roubicek DA, de Souza-Pinto NC. Mitochondria and mitochondrial DNA as relevant targets for environmental contaminants. Toxicology. 2017;391:100-8.

57. Munot K, Bell SM, Lane S, Horgan K, Hanby AM, Speirs V. Pattern of expression of genes linked to epigenetic silencing in human breast cancer. Hum Pathol. 2006;37:989-99.

58. Yu M, Zhou Y, Shi Y, Ning L, Yang Y, Wei X, et al. Reduced mitochondrial DNA copy number is correlated with tumor progression and prognosis in Chinese breast cancer patients. IUBMB Life. 2007:59:450-7.

59. Timinskas A, Au Z, Ku V. Atherosclerosis: alterations in cell communication; 2007. p. 6.

60. Bayraktar G, Yuanxiang P, Confettura AD, Gomes GM, Raza SA, Stork O, et al. Synaptic control of DNA methylation involves activity-dependent degradation of DNMT3A1 in the nucleus. Neuropsychopharmacology. Nature Publishing Group. 2020. p. 1-11.

61. Guantes R, Rastrojo A, Neves R, Lima A, Aguado B, Iborra FJ. Global variability in gene expression and alternative splicing is modulated by mitochondrial content. Genome Res. 2015;25:633-44.
62. Ulrey $C L$, Liu L, Andrews LG, Tollefsbol TO. The impact of metabolism on DNA methylation. Hum Mol Genet. Oxford Academic. 2005;14:R139-47.

63. Day JJ, Kennedy AJ, Sweatt JD. DNA methylation and its implications and accessibility for neuropsychiatric therapeutics. Annu Rev Pharmacol Toxicol. 2015;55:591-611.

64. Hu L, Li Z, Cheng J, Rao Q, Gong W, Liu M, et al. Crystal structure of TET2DNA complex: insight into TET-mediated 5mC oxidation. Cell. 2013;155: 1545-55.

\section{Publisher's Note}

Springer Nature remains neutral with regard to jurisdictional claims in published maps and institutional affiliations.
Ready to submit your research? Choose BMC and benefit from:

- fast, convenient online submission

- thorough peer review by experienced researchers in your field

- rapid publication on acceptance

- support for research data, including large and complex data types

- gold Open Access which fosters wider collaboration and increased citations

- maximum visibility for your research: over $100 \mathrm{M}$ website views per year

At BMC, research is always in progress.

Learn more biomedcentral.com/submissions 